\title{
Total Block Edge Cut Vertex Graph
}

\author{
Venkanagouda M Goudar \\ Sri Gouthama Research centre (Affiliated to Kuvempu University), Department of \\ Mathematics, Sri Siddhartha Institute of Technology \\ Email:vmgouda@gmail.com
}

\begin{abstract}
In this paper, we introduce the concept of the total block edge cut vertex graph. We obtain some properties of these graphs. We present characterization of graphs whose total block edge cut vertex graphs planar, outer planar, minimally non outer planar and crossing number one.
\end{abstract}

Keywords: Block, cutvertex, cutvertex graph, edge, line graph.

\section{Introduction}

By a graph, we mean a finite, undirected graph without loops or multiple edges. Definitions not given here may be found in [1]. We now define the total block edge cut vertex graph. For any graph $G$ with cut vertex set $C(G)$, edge set $E(G)$ and block set $B(G)$. The total block edge cut vertex graph $B^{* *}(G)$ of $G$ is defined as the graph having vertex set $C(G) \cup E(G) \cup B(G)$, with two vertices adjacent if they correspond to two adjacent edges of $\mathrm{G}$ or one corresponds to an edge of $\mathrm{G}$ and other to a block of $\mathrm{G}$ and $\mathrm{e}_{\mathrm{i}}$ lies in $\mathrm{B}_{\mathrm{j}}$ or one corresponds to a block and other to a cut vertex $c_{j}$ of $G$ and $c_{j}$ lies on $B_{j}$ or both cut vertices are adjacent.

\section{Preliminary Results}

Theorem 2.1 [1] If $G$ is a (p, q) graph whose vertices have degree $d_{i}$ then $L(G)$ has q vertices and $\mathrm{q}_{\mathrm{L}}$ edges where $q_{L}=1 / 2 \sum_{i=1}^{p} d_{i}^{2}$.

Theorem 2.2 [2] The line graph $\mathrm{L}(\mathrm{G})$ of a graph is planar if and only if $\mathrm{G}$ is planar, $\Delta(\mathrm{G}) \leq 4$ and if deg $\mathrm{v}=4$ for a vertex $\mathrm{v}$ of $\mathrm{G}$, then $\mathrm{v}$ is a cut vertex. 
Theorem 2.3 [3] A graph is planar if and only if it has no sub graph homeomorphic to $\mathrm{K}_{5}$ or $\mathrm{K}_{3,3}$.

Theorem 2.4 [ 3] A graph is outer planar if and only if it has no sub graph homeomorphic to $\mathrm{K}_{4}$ or $\mathrm{K}_{2,3}$.

Theorem 2.5 [5] The block edge cut vertex graph $B^{*}(G)$ of a graph $G$ has crossing number one if and only if $G$ is planar and satisfies the following conditions.

1. Degree of every vertex is at most 4 and

2. G has a unique vertex of degree 4 which is a cut vertex or

3. in $\mathrm{G}$ any one block with exactly two non cut vertices of degree 3 which are adjacent and all other vertices of degree at most 3 .

The following observations are of use.

Lemma 2.1 For any graph $G$, the line graph $L(G)$ is a sub graph of $B^{* *}(G)$.

Lemma 2.2 If $G$ is a cycle $C_{n}$, then $B *(G)$ becomes a wheel $W_{n}$.

Lemma 2.3 If $K_{1, p}, p \geq 2$ having a cut vertex of degree $p$ is a sub graph of $G$ then $\mathrm{K}_{\mathrm{p}}$ is a sub graph of $\mathrm{B}^{* *}(\mathrm{G})$.

Lemma 2.4 If $K_{1, p}, p \geq 2$ having a non cut vertex of degree $p$ is a sub graph of $\mathrm{G}$ then $\mathrm{K}_{\mathrm{p}+1}$ is a sub graph of $\mathrm{B}^{* *}(\mathrm{G})$.

\section{Main Results.}

The first theorem determines the number of vertices and edges in the total block edge cut vertex graph of a graph.

Theorem 3.1 If $\mathrm{G}$ is nontrivial connected (p, q) graph with b block and k cut vertices, whose vertices have degree $d_{i}, l_{i}$ be the number of edges to which block $b_{i}$ belongs and $n_{i}$ be the number of blocks to which the cut vertex $c_{i}$ belongs in $G$, then the total block edge cut vertex graph $\mathrm{B}^{* *}(\mathrm{G})$ has $(\mathrm{q}+\mathrm{b}+\mathrm{k})$ vertices and $\frac{1}{2} \sum_{i=1}^{p} d_{i}^{2}+\sum_{i=1}^{b} n_{i}+\sum_{i}^{k} \frac{c_{i}\left(c_{i}-1\right)}{2}$ edges.

Proof. By definition of total block edge cut vertex graph $B^{* *}(G)$, it has $(q+b$ $+\mathrm{k})$ vertices. The number of edges of $B^{* *}(G)$ is the sum of the number of edges in $\mathrm{L}(\mathrm{G})$, the number of edges in each block, the number of blocks incident to cut vertices $n_{i}$ of $G$ and the number of edges in a cut vertex graph. Now $G$ has q edges 
and by Theorem 2.1, $\mathrm{L}(\mathrm{G})$ has $-\mathrm{q}+\frac{1}{2} \sum d_{i}{ }^{2}$ edges. Also the sum of the edges incident to block is $\sum q_{i}=\mathrm{q}$ and $E[C(G)]=\sum_{i}^{k} \frac{c_{i}\left(c_{i}-1\right)}{2}$. Hence the number of edges in $\mathrm{B}^{* *}(\mathrm{G})=-q+\frac{1}{2} \sum_{i=1}^{p} d_{i}^{2}+q+\sum_{i=1}^{b} n_{i}+\sum \frac{c_{i}\left(c_{i}-1\right)}{2}$

$$
=\frac{1}{2} \sum_{i=1}^{p} d_{i}^{2}+\sum_{i=1}^{b} n_{i}+\sum_{i}^{k} \frac{c_{i}\left(c_{i}-1\right)}{2} \text { edges. }
$$

Theorem 3.2 Let $G$ be a nontrivial connected (p, q) graph. The graphs $G$ and $\mathrm{B}^{* *}(\mathrm{G})$ are isomorphic if and only if $\mathrm{G}=\mathrm{K}_{2}$.

Proof . Suppose $\mathrm{G}$ is isomorphic $\mathrm{B}^{* *}(\mathrm{G})$. Assume $\mathrm{G}$ is a connected graph with $\mathrm{p}$ $\geq 3$. We consider the following cases:

case 1. Suppose $G$ is not a tree with $p$ vertices. Then it has at least $p$ edges and has at least one block. Thus $B^{*}(G)$ has at least $p+1$ vertices. Hence the number of vertices in $G$ is less than that in $B^{* *}(G)$. Then $G \neq B^{* *}(G)$, a contradiction.

case 2. Suppose $G$ is a tree with $p$ vertices. Then it has $p-1$ edges and $p-1$ blocks. Thus $B^{* *}(G)$ has $2 p-2+k$ vertices where $k$ is the number of cut vertices. Hence the number of vertices of $G$ is less than that is $B^{*}(G)$. Thus the graphs $G$ and $\mathrm{B} * *(\mathrm{G})$ are not isomorphic, a contradiction.

In each case we arrive a contradiction. Thus, $\mathrm{G} \cong \mathrm{K}_{2}$.

Theorem 3.3 The total block edge cut vertex graph $\mathrm{B}^{* *}(\mathrm{G})$ and the block edge cut vertex graph $B *(G)$ are isomorphic if and only if $G$ has at most one cut vertex.

Proof. Suppose $B^{*}(G) \cong B^{* *}(G)$.From the Theorem 2.1 and the Theorem 3.1, the graph $\mathrm{B}^{*}(\mathrm{G})$ and $\mathrm{B}^{* *}(\mathrm{G})$ have $\frac{1}{2} \sum d_{i}^{2}+\sum n_{i}$ and $\frac{1}{2} \sum d_{i}^{2}+\sum n_{i}+\sum \frac{c_{i}\left(c_{i}-1\right)}{2}$ edges respectively. Since $\mathrm{B}^{*}(\mathrm{G}) \cong \mathrm{B}^{* *}(\mathrm{G})$, this implies that $\sum \frac{c_{i}\left(c_{i}-1\right)}{2}=0$. $\Rightarrow c_{i}=0, c_{i}=1$.

If $c_{i}=0$, then $\mathrm{G}$ has no cut vertex, since $\mathrm{G}$ is connected and it must be a block. If $c_{i}=1$, then $\mathrm{G}$ has only end blocks. This implies that $\mathrm{G}$ has at most one cut vertex.

Conversely, suppose $\mathrm{G}$ has at most one cut vertex. We consider the following the cases:

Case ( $\mathbf{i}$ ). Suppose $G$ has no cut vertex. Then $G$ is a block. Obviously $B^{*}(G)$ and $\mathrm{B} * *(\mathrm{G})$ have the same number of vertices and edges. 
Case (ii). Suppose $\mathrm{G}$ has a cut vertex. Let it be incident with more than two edges. Then the cut vertex together with its $\mathrm{n}$ incident edges forms $\left\langle K_{n+1}\right\rangle$ has a sub graph of $B^{*}(G)$ and hence in $B^{* *}(G)$. Hence $B^{*}(G) \cong B^{* *}(G)$.

Theorem 3.4. The total block edge cut vertex graph $B * *(G)$ of a graph $G$ is planar if and only if $\mathrm{G}$ satisfies the following conditions:

i). $\Delta(G) \leq 3$

ii). every block of $\mathrm{G}$ is either a cycle or $\mathrm{K}_{2}$.

iii). each nontrivial block contains nonadjacent cut vertices.

Proof. Suppose the total block edge cut vertex graph $\mathrm{B}^{* *}(\mathrm{G})$ is planar. By lemma $2.1 \mathrm{~L}(\mathrm{G})$ is planar. By Theorem $2.2, \Delta(G) \leq 4$.

Suppose $\Delta(G)=4$. Then there exist a vertex $\mathrm{v}$ in $\mathrm{G}$ such that deg (v) $=4$. By Theorem 2.2, $\mathrm{v}$ is a cut vertex . The four incident edges of the cut vertex form $\mathrm{K}_{4}$ as a sub graph of $\mathrm{B}^{* *}(\mathrm{G})$ in which all the edges are interior and hence it is a non planar, a contradiction. Thus no vertex of $\mathrm{G}$ has degree 4 . Hence, $\Delta(G) \leq 3$. This proves (i).

Assume that $G$ is a block which is neither an edge nor a cycle. Clearly $G$ contains at least five edges. The line graph $\mathrm{L}(\mathrm{B})$ of $\mathrm{B}$ has a sub graph of $\mathrm{K}_{4}-\mathrm{x}$, where $\mathrm{x}$ is any edge of $\mathrm{K}_{4}$. The vertex in $\mathrm{B}^{* *}(\mathrm{G})$ which corresponds to $\mathrm{B}$ is adjacent to every vertex of $\mathrm{L}(\mathrm{B})$. This produces a sub graph homeomorphic to $\mathrm{K}_{6}$ $-2 \mathrm{x}$ in $\mathrm{B}^{* *}(\mathrm{G})$, where $\mathrm{x}$ is any edge of $\mathrm{K}_{6}$, which is non planar, a contradiction. Hence both conditions are satisfied.

Further, we assume that each nontrivial block contains at least two adjacent cut vertices. Thus the cut vertices together with edge of block form $\mathrm{K}_{5}$ as a sub graph , which is non planar, contradiction. Hence all conditions are satisfied.

Conversely, suppose $\mathrm{G}$ satisfies all conditions of the theorem .Clearly every block of $G$ is either a cycle or a $K_{2}$. It is easy to see that $G$ is planar and by Theorem 2.2, $\mathrm{L}(\mathrm{G})$ is planar. By lemma 2.1, $\mathrm{L}(\mathrm{G})$ is a sub graph B**(G). Assume that a cycle $C_{r}$ is a block of $G$. Then $L\left(C_{r}\right)$ is a cycle in the planar graph of $L(G)$. Since in the planar drawing of $\mathrm{L}(\mathrm{G})$ every such cycle can be drawn with all its vertices on the boundary of one region, consider a crossing -free drawing of $L(G)$ in which all vertices of the sub graph $\mathrm{L}\left(\mathrm{C}_{\mathrm{r}}\right)$ are on the boundary of one region. Then the vertex $u_{r}$ corresponding to the block $C_{r}$ of the graph $G$ can be placed into this region in such a way that the edges joining $u_{r}$ with the vertices of $L\left(c_{r}\right)$ do not cross the edges of $L(G)$. Next assume that $K_{2}$ is a block of $G$. Then $L\left(K_{2}\right)$ is the vertex say $w_{n}$ in $L(G)$. Let $u_{n}$ be the vertex in $B^{*}(G)$ corresponding to the block $K_{2}$ of $G$. Then the edge $\left\{u_{n} W_{n}\right\}$ is in $B^{* *}(G)$. This edge can be placed in some region of $\mathrm{B}^{* *}(\mathrm{G})$ without losing planarity. Also the vertex $\mathrm{v}$ in $\mathrm{B} *(\mathrm{G})$ corresponding to the cut vertex of $G$ and the edges joining $v$ with the vertex $w_{n}$ do not cross the edges in $\mathrm{B}^{* *}(\mathrm{G})$. Thus $\mathrm{B}^{* *}(\mathrm{G})$ is planar. This completes the proof. 
Theorem 3.5 The total block edge cut vertex graph $B^{* *}(G)$ is outer planar if and only $\mathrm{G}$ is a path $\mathrm{P}_{3}$.

Proof Suppose $\mathrm{B}^{* *}(\mathrm{G})$ is outer planar. Cleary $\mathrm{B}^{*}(\mathrm{G})$ is planar, By Theorem 3.1, $\Delta=3$ and $\mathrm{G}$ is either a cycle or $\mathrm{K}_{2}$. Assume $\Delta(G)=3$ and $\mathrm{v}$ is a vertex of $\mathrm{G}$ with degree three. If $v$ is not a cut vertex, then it self is a block $b$. Then number of edges in a block is at least five. These five edges together with $b$ form $\left\{K_{6}-2 x\right\}$ is a sub graph of $\mathrm{B}^{* *}(\mathrm{G})$, which is non planar, a contradiction. Also if $\mathrm{v}$ is a cut vertex, then it form $\mathrm{K}_{5}$ as a sub graph which is non planar, a contradiction.

Assume that $\mathrm{G}$ is not a path. We consider the following cases.

Case1. Suppose $\mathrm{G}$ is a cycle. Then by Theorem 4, B**(G) is a wheel, which is non outer planar, a contradiction.

Case 2. Suppose $G$ is a star $K_{1,3}$. Then $L(G)$ is a $K_{3}$. Also each edge is a block. Every vertex of $L(G)$ is joins a vertex $b_{1}, b_{2}, b_{3}$. Then $b_{i}$ together with cut vertex $\mathrm{v}$ form a graph which is homeomorphic to $\mathrm{K}_{4}$. By Theorem 2.4 , B**(G) is non outer planar, a contradiction. Hence $G$ must be a path $P_{n}$ for $n<4$.

Conversely suppose $G$ is a path $P_{n}$ for $n<4$. Then every block of $B * *(G)$ is a cycle $\mathrm{C}_{5}$. Hence $\mathrm{B}^{* *}(\mathrm{G})$ is outer planar. This completes the proof.

Theorem 3.6 The total Block edge cut vertex graph $B^{*}(G)$ of $G$ is minimally non outer planar if and only if $\mathrm{G}$ is a path $\mathrm{P}_{4}$.

Proof. Suppose $B^{*}(G)$ is minimally non outer planar. Assume that $G$ is not a path $\mathrm{P}_{4}$. We consider the following cases:

Case 1. $G$ is a block. Clearly it contains at least five edges. Then the $L(G)$ together with block $b$ forms a graph $\left\{\mathrm{K}_{6}-2 \mathrm{x}\right\}$ which has $\mathrm{i}\left(\mathrm{B}^{* *} \mathrm{G}\right)>2$, a contradiction.

Case 2. Assume that $G$ contains at least one cut vertex of degree 3. Then $L(G)$ together with cut vertex $c$ and block $b$ form at least two $\left\{K_{4}\right\}$ as induced sub graphs in $\mathrm{B}^{* *}(\mathrm{G})$. Thus $\mathrm{i}\left\{\mathrm{B}^{* *}(\mathrm{G})\right\}>1$, a contradiction.

Case 3. Assume that $G$ is a path $P_{n}$ for $n>4$. If $n=5$, then $L\left[P_{5}\right]=P_{4}$ and each block is an edge and each cut vertex incident with two edges. Also $C\left(\mathrm{P}_{4}\right)$ is a path $\mathrm{P}_{3}$. Clearly $\mathrm{i}\left[\mathrm{B} * *\left(\mathrm{P}_{5}\right)\right]=2$, a contradiction.. Hence $\mathrm{G}=\mathrm{P}_{4}$.

Conversely suppose $\mathrm{G}$ is a cycle $\mathrm{C}_{\mathrm{n}}$. By the definition, $\mathrm{L}(\mathrm{G})$ together with block $\mathrm{b}$ form a wheel $\mathrm{W}_{\mathrm{n}}$. It is known that every wheel is minimally non outer planar. This completes the proof.

Theorem 3.7 The total block edge cut vertex graph $B^{* *}(G)$ of a graph $G$ has crossing number one if and only if $G$ is planar and satisfies the following conditions.

i). Degree of every vertex is at most 4 and

ii). $G$ has a unique vertex of degree 4 which is a cut vertex or

iii). In $G$ any one block with exactly two non cut vertices of degree 3 which are adjacent and all other vertices of degree at most 3 . 
Proof. Proof follows from the Theorem 2.5.

\section{Conclusion.}

In this paper we presented the total block edge cut vertex graph of a graph. Also we presented the planarity, outer planarity and crossing number one.

\section{References}

[1] Haray. F, Annals of New York, Academy of Sciences, (1977).

[2] Harrary.F, Graph Theory, Addison- Wesley Reading Mass, (1969).

[3] Kulli. V R, On minimally non outer planar graphs, proceedings of the Indian National Science Academy, 41, (1975).

[4] Sedlacek J., Some properties of interchange graphs. The Graphs and the applications. Academic press, New York, (1962).

[5] Y.B.Maralabhavi, Venkanagouda. M. Goudar., Block edge cutvertex graph, Acta Ciencia Vol.XXXIII M. No. 2. 493, (2007). 